\title{
Editorial
}

\section{Artificial Intelligence or Clinical Intelligence for Better Health}

\author{
Rajeev Gupta \\ Editor, RUHS Journal of Health Sciences; Chair, Academic and Research Development Unit, \\ Rajasthan University of Health Sciences, Jaipur, Rajasthan, India \\ DOI:10.37821/ruhsjhs.5.1.2020.3-5
}

Poor healthcare access and inconsistent quality of care is widespread in India and most low and lower-middle income countries. ${ }^{1}$ Difficulty in adequately training healthcare providers including physicians, is an important challenge. ${ }^{2}$ Other challenges include inadequacy of systems for ensuring that healthcare providers (physicians, nurses, technicians, and other paramedics) have access to rapidly changing clinical information and management standards and paucity of efficient and robust data-driven technologies for guiding clinical decision making. ${ }^{3}$ In absence of these inputs physicians and other healthcare workers rely on centuries old techniques of didactic learning for gathering information to improve clinical practice and patient outcomes. ${ }^{4}$

\section{ARTIFICIAL INTELLIGENCE}

It has been suggested that artificial intelligence (AI) has the potential to revolutionize healthcare and health not only in India and other low and lower-middle income countries but also in high-income countries. ${ }^{3,5}$ AI powered virtual assistants, predictive algorithms, customized care-plans, and home-based nudges can be game-changing for delivering care to those with little to no care. ${ }^{6}$ However, deep neural networks, the complex, multi-layered, self-teaching models that are expected to eventually aid and nudge physicians, will only be as good as the data that power them. ${ }^{7}$ The more structured and labeled the data, the readier they are for machines to ingest them. The most successful inroads we have therefore seen are those where the data are the cleanest, for example in radiology images or in pathology slides. ${ }^{8}$ However, in order for this vision to be rolled out globally to reach the billions that do not have the most basic access to care we need more than Silicon Valley hubris and Dalal Street speculation.

A number of focus areas for AI in health exist. These vary from estimation of global, national, and subnational burden of disease using multiple data sources and Bayesian metaregression techniques driven by deep learning algorithms, ${ }^{9}$ to disease diagnosis using multiple predictive algorithms powered by convolutional neural networks, ${ }^{10}$ and precision medicine predictions for an individual patient using multiple layers of omics information ranging from DNA genomics to planetary environmentonomics. ${ }^{11,12}$ A number of studies have evaluated applications of AI in healthcare such as algorithms for interpreting chest radiographs, detecting cancer in mammograms, analyzing computer tomography scans, identifying brain tumors on magnetic resonance images, and predicting development of Alzheimer's disease from positron emission tomography. ${ }^{13} \mathrm{AI}$ has also been evaluated in pathology, identifying cancerous skin lesions, interpreting retinal imaging for various disease conditions including atherosclerosis, detecting arrhythmias, and identifying hyperkalemia from electrocardiograms. ${ }^{13}$ It has also aided in polyp detection from colonoscopy, improving genomics interpretation, and identifying genetic conditions from facial appearance.

However, there is need for robust clinical evaluation, using metrics that are intuitive to clinicians and ideally go beyond measures of technical accuracy to include quality of care and patient outcomes. ${ }^{7}$ Further work is required to identify themes of algorithmic bias and unfairness while developing mitigations to address these, to reduce brittleness and improve generalizability, and to develop methods for improved interpretability of machine learning predictions. ${ }^{13}$ A number of clinical trials have compared outcomes using either AI based algorithms or standard clinical methods. In no trial AI has passed the test of equivalence leave alone superiority. ${ }^{12}$ The major limitation of the AI based diagnostic and therapeutic outcome is the quality of data used for machine learning with the biggest challenge being the quality of the input data. ${ }^{14}$ Garbage in 
Garbage out aphorism fits perfectly with the current status of AI in medicine.

\section{CLINICAL INTELLIGENCE}

Clinical intelligence, on the other hand, is based on synthesis of hard data obtained by clinical history and examination and lots of soft data such as listening to the patient, reading between the lines and understanding posturing and mannerisms. AI cannot obtain the soft data and interpretation of such data is presently beyond its realm.

An important use of AI has been in disease detection. Liu et $\mathrm{al}^{15}$ compared deep learning performance against healthcare professionals in detecting disease from medical imaging. Eighty two studies describing 147 patient cohorts were identified of which 679 studies provided enough data to construct contingency tables. Sensitivity of AI to diagnose abnormality ranged from 9.7-100.0\% (mean $79.1 \pm 0.2)$ while specificity ranged from $38.9-100.0 \%$ (mean 88.3 \pm 0.1 ). Although this meta-analysis found that diagnostic performance of deep learning models was equivalent to that of health-care professionals the major limitation was that only a few studies presented externally validated results or compared the performance of deep learning models and health-care professionals using the same sample. Quality of data used in many of these studies was sub-optimal. A number of decision support systems are being developed to guide practice. ${ }^{16}$ Many teams are in the midst of extensive research regarding potential machine learning solutions but only a handful have received US FDA approval (Table). ${ }^{11,12}$ This number is rising rapidly.

Table: Selected list of US FDA approved artificial intelligence platforms

\begin{tabular}{ll}
\hline Company & \multicolumn{1}{c}{ Indication } \\
\hline Apple & Atrial fibrillation diagnosis \\
Aidoc & CT brain bleed diagnosis \\
iCAD & Breast density via mammography \\
Zebra Medical & Coronary calcium scoring \\
Bay Labs & Echocardiogram EF determination \\
Neural Analytics & Device for paramedic stroke diagnosis \\
Idx & Diabetic retinopathy diagnosis \\
Icometrix & MRI brain interpretation \\
Imagen & X-ray wrist fracture diagnosis \\
Viz.ai & CT stroke diagnosis \\
Artesys & Liver and lung cancer (CT, MRI) diagnosis \\
MaxQ-AI & CT brain bled diagnosis \\
Alivecor & Atrial fibrillation detection via Apple Watch \\
Artesys & MRI heart interpretation \\
\hline
\end{tabular}

On the other hand, only a few randomized clinical trials of artificial intelligence exist. In a trial to assess AI based hypotension prediction during surgery tool versus usual clinical care, the tool was found superior to usual care with less time-weighted average of intraoperative hypotension episodes and fewer episodes of hypotension. ${ }^{17}$ Decision support systems have been developed for diagnosis of management of hypertension and diabetes in low income countries but have not been uniformly successful. A systematic review concluded that technological interventions using algorithm based (AI) management was only successful when implemented as a part of multicomponent interventions. ${ }^{18}$

Mehta et $\mathrm{al}^{5}$ suggest that for AI to fulfill its promise to promote global health three key challenges need to be addressed: (i) reliability and availability of high quality health related data; (ii) development and application of context-specific AI tools; and (iii) regulatory capacity to oversee and manage the rapidly changing technology. These obstacles can be tackled with large investments in data collection, standardization and availability in resource poor settings using low cost technologies. Industries building AI-driven solutions can be enticed to build and validate their solutions for low and lower-middle countries contexts. Healthcare workers would also need to be trained to appropriately use these technologies and public sector should invest in regulatory capacity. ${ }^{5}$ Billions shall have to be spent in creating such an AI network and whether such AI-tools perform optimally shall have to be validated in clinical trials. ${ }^{12,19}$

The father of modern medicine Sir William Osler has famously said- he who studies medicine without books sails an uncharted sea, but he who studies without patients does not go to sea at all. ${ }^{20} \mathrm{AI}$ is still in the stage of evolution (sailing an uncharted sea) and till the time it is truly evolved and converts itself into an empathetic and compassionate being, clinical intelligence shall continue to be better. Francis Peabody's century old aphorism- healthcare after all is humans caring for humans-still rings true. ${ }^{21}$

\section{REFERENCES}

1. Kruk M, Gage AD, Arsenault C, Jordan K, Leslie H, Dewan SR et al. High quality health systems: Time for a revolution. Lancet Glob Health. 2018; 6:e1196-1252. 
2. Gupta R. Quality issues in primary healthcare. RUHS $J$ Health Sciences. 2018; 3:3-4.

3. Institute of Medicine. Crossing the global quality chasm: Improving healthcare worldwide. Washington DC: National Academies Press. 2018.

4. Gupta R. Creating health professionals for the $21^{\text {st }}$ century. RUHSJ Health Sciences. 2017; 2:161-62.

5. Mehta MC, Katz IT, Jha AK. Transforming global health with AI. NEnglJ Med. 2020;382:791-93.

6. Balsari S. Will AI help universalize health care? BMJ Blogs.2019. Available at: https://blogs.bmj.com/bmj/2019/ 09/23/satchit-balsari-will-ai-help-universalize-healthcare/. Accessed 15 March 2020.

7. Mitchell M. Artificial intelligence: A guide for thinking humans. New York. Farrar, Straus and Giroux. 2019.

8. Miller DD. The medical AI insurgency: What physicians must know about data to practice with intelligent machines. NPJDigit Med. 2019; 2:1-5.

9. Flaxman AD, Vos T, Murray CJL. An integrative metaregression framework for descriptive epidemiology. Seattle. University of Washington Press. 2015.

10. Editorial. Walking the tightrope of artificial intelligence guidelines in clinical practice. Lancet Digit Health. 2019; 1:e100.

11. Topol EJ. High performance medicine: The convergence of human and artificial intelligence. Nature Med. 2019; 25:4456.

12. Topol EJ. Deep Medicine. New York. Basic Books, 2019.

13. Kelly CJ, Karthikesalingam A, Suleyman M, Corrado G, King D. Key challenges for delivering clinical impact with artificial intelligence. BMCMed.2019; 17:e195.

14. Riley RD, Ensor J, Snell KIE, Harrel FE, Martin GP, Reitsma JB et al. Calculating the sample size required for developing a clinical prediction model. BMJ.2020;368: $\mathrm{m} 441$.

15. Liu X, Faes L, Kale AU, Wagner SK, Fu DJ, Bruynseels A et al. A comparison of deep learning performance against health-care professionals in detecting diseases from medical imaging: a systematic review and meta-analysis. Lancet Dig Health. 2019; 1:e271-97.

16. Angus DC. Randomized clinical trials of artificial intelligence. JAMA. 2020;323:1043-45.

17. Wijnberge M, Geerts BF, Hol L,Lemmers N, Mulder PM, Berge P, Schenk J et al. Effect of a machine learning derived early warning system for intraoperative hypotension vs standard care on depth and duration of intraoperative hypotension during elective noncardiac surgery: The HYPE randomized clinical trial. JAMA.2020;323:1052-60.

18. Correia JC, Lachat S, Lagger G, Chappuis F, Golay A, Beran $\mathrm{D}$ et al. Interventions targeting hypertension and diabetes mellitus at community and primary healthcare level in lowand middle-income countries: A scoping review. $B M C$ Public Health. 2019; 19:1542.

19. Wild HB. There's no algorithm for empathy: A personal story of cancer, medical education, physicians, and clinical empathy. Health Aff (Milwood). 2020;39:339-42.

20. Osler W. William Osler quotes. Available at: https://www. brainyquote.com/authors/william-osler-quotes. Accessed 20 March 2020.

21. Peabody FW. The care of the patient. JAMA. 1927; 88:87782. 